\title{
Expression study of the target receptor tyrosine kinase of Imatinib mesylate in skull base chordomas
}

\author{
FRANCESCA ORZAN ${ }^{1}$, MARIA ROSA TERRENI ${ }^{2}$, MAURO LONGONI $^{1}$, NICOLA BOARI $^{3}$, \\ PIETRO MORTINI ${ }^{4}$, CLAUDIO DOGLIONI ${ }^{2}$ and PAOLA RIVA ${ }^{1}$ \\ ${ }^{1}$ Department of Biology and Genetics, Medical Faculty, University of Milan, Via Viotti 3/5, 20133 Milano; \\ Departments of ${ }^{2}$ Pathology, and ${ }^{3}$ Neurosurgery, S. Raffaele Scientific Institute, via Olgettina 60, 20132 \\ Milano; ${ }^{4}$ Department of Neurosurgery, University of Brescia, Viale Europa 11, 25133 Brescia, Italy
}

Received February 5, 2007; Accepted March 20, 2007

\begin{abstract}
Chordomas are rare neoplasms arising along the axial skeleton. Up to now, the most suitable therapeutic approach is based on a combination of surgical excision and radiotherapy. Chemotherapy in not applied due to its reported low efficacy. Recently, evidence on the efficacy of Imatinib mesylate in two patients has been reported. We analyzed 14 chordoma samples for the expression of the Imatinib mesylate targets by means of RT-PCR and immunohistochemistry and found that PDGFR $\alpha$ and PDGFRß are in some cases expressed in neoplastic cells, while the stromal counterpart of the same tumor shows the above receptors. Findings on the PDGFA/ $P D G F B$ expression suggest a receptor-activated status. Our study provides new insights into the specific localization of Imatinib mesylate targets in skull base chordomas that could be taken into account for the setting up of a pharmacological treatment for this tumor.
\end{abstract}

\section{Introduction}

Chordomas are rare slow-growing neoplasms of the axial skeleton with two main sites of origin, the sacrococcigeal region and the skull base (clivus). The treatment of chordomas is still controversial. Surgical excision followed by radiotherapy, in particular proton beam irradiation, is related with the best tumor progression-free survival (1). Chemotherapy is not currently applied in clinical practice due to the described low efficacy (2-5). The course of the disease is often unfavorable due to local recurrence and eventual death. Recently, Casali et al reported on the compassionate use of

Correspondence to: Dr Paola Riva, Department of Biology and Genetics, Medical Faculty, University of Milan, Via Viotti 3/5, 20133 Milano, Italy

E-mail: paola.riva@unimi.it

Key words: skull base chordoma, PDGFR, KIT, Imatinib mesylate
Imatinib mesylate in 6 patients affected by chordoma ( 1 skull base and 5 sacral chordomas), 4 of which expressed PDGFRß (6). The authors observed tumor colliquation in one patient and a decrease in tumor density in another one. The study revealed an antitumor activity of Imatinib mesylate. As this drug exerts its activity by inactivating PDGFR $\beta$, PDGFR $\alpha$ and c-kit, it is important to investigate the expression of these receptors in a higher number of chordomas. We therefore evaluated the expression of PDGFRA, PDGFRB and KIT transcripts and proteins and the mRNA expression of their ligands, $P D G F A, P D G F B$ and $S C F$, in 14 skull base chordoma (SBC) samples.

\section{Materials and methods}

Tumor samples. The study was performed on 13 SBC samples and on a recurrence from one of them. Each patient underwent surgery at the Department of Neurosurgery of the San Raffaele Scientific Institute in Milan between August 1997 to December 2005. Nine patients were male and four were female; their ages ranged from 25 to 67 years (average 40,5 years). All the patients had been treated with radiotherapy. All the patients gave written informed consent in order to participate in the study.

RT-PCR analysis. The expression analysis of the Imatinib targets was performed by RT-PCR. RNA isolated from 14 fresh or frozen chordoma samples was carried out by means of the TRIzol reagent (Invitrogen, Carlsbad, CA, USA); $1 \mu \mathrm{g}$ of total RNA was reverse transcribed using the ThermoScript RT-PCR System and oligo-dT primers for first-strand cDNA synthesis according to the instructions of the manufacturer (Invitrogen). Specific primers for KIT, PDGFRA and PDGFRB were designed on different exons for each amplimer (Table I); the ligand primers were synthesized according to Matei et al (7). cDNAs were analyzed by agarose gel electrophoresis.

Immunohistochemistry. Immunohistochemical analysis was performed on formalin-fixed paraffin-embedded material. In 12 cases adequate histological material was available. The following antibodies were utilized: a rabbit polyclonal PDGF Receptor alpha Antibody (cod.3164, Cell Signaling 
Table I. KIT, PDGFRA and PDGFRB primer sequences and PCR conditions.

\begin{tabular}{lcccc}
\hline Gene & Forward primer & Reverse primer & \multicolumn{2}{c}{ PCR conditions } \\
\hline KIT & GATCCCATCGCAGCTAC & TCATATAGATCCACTGCTG & $95^{\circ}-62^{\circ}-72^{\circ}$ & 10 cycles \\
& & & $30^{\prime \prime}-30^{\prime \prime}-30^{\prime \prime}$ & \\
& & & $95^{\circ}-56^{\circ}-72^{\circ}$ & 35 cycles \\
& & & $30^{\prime \prime}-30^{\prime \prime}-30^{\prime \prime}$ & \\
PDGFRA & \multirow{2}{*}{ GGGGAAACGATTGTGGTCACC } & CCCGCACCTCTACAACAAAAT & $95^{\circ}-62^{\circ}-72^{\circ}$ & 40 cycles \\
& & & $30^{\prime \prime}-30^{\prime \prime}-30^{\prime \prime}$ & \\
PDGFRB & \multirow{2}{*}{ GATTCTGATGCCTACTATGTC } & CAGGGTGCGGTTGTCTTGA & $95^{\circ}-62^{\circ}-72^{\circ}$ & 5 cycles \\
& & & $30^{\prime \prime}-30^{\prime \prime}-25^{\prime \prime}$ & \\
& & & $95^{\circ}-58^{\circ}-72^{\circ}$ & 35 cycles \\
& & & $30^{\prime \prime}-30^{\prime \prime}-30^{\prime \prime}$ & \\
\hline
\end{tabular}

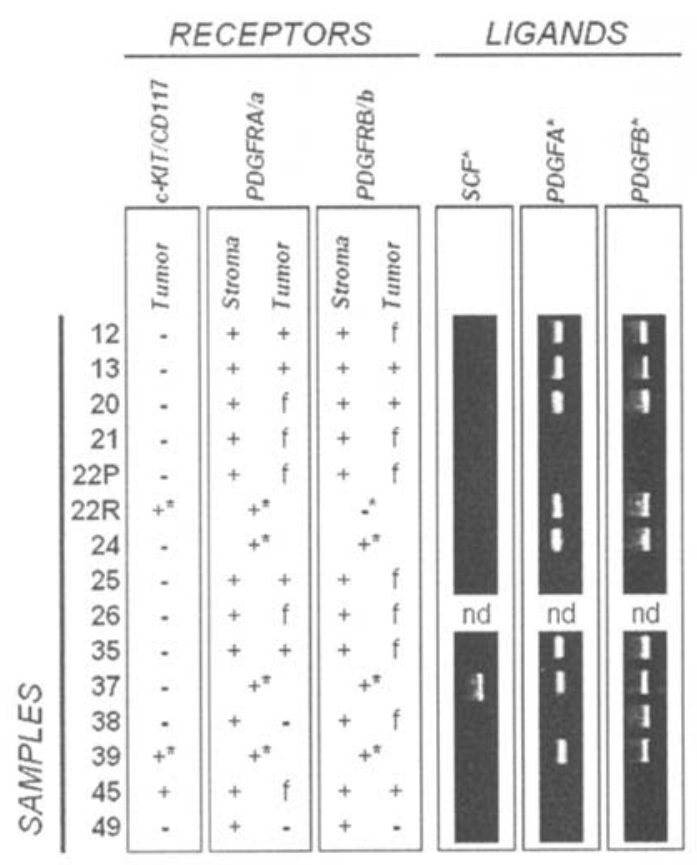

Figure 1. Expression of c-kit, PDGFR $\alpha$, PDGFR $\beta$ and their ligands, $S C F$, $P D G F A$ and $P D G F B$, in 15 chordoma samples by immunohistochemical analysis. +, Positive; -, negative; f, focal expression; *data obtained by RTPCR.

Technology, Danvers, MA, USA) diluted 1/100; a rabbit monoclonal PDGF Receptor beta Antibody (cod.3169, Cell Signaling Technology) diluted $1 / 50$; and a rabbit polyclonal antibody against c-kit (cod.A4502, Dako, Glostrup, Denmark), diluted 1/500. Briefly, the histological sections were deparaffinized, rehydrated and endogenous peroxidase activity was quenced with $3 \%$ hydrogen peroxide. Tris buffer $0.05 \mathrm{M}$ with EDTA $0.01 \mathrm{M}$ at $\mathrm{pH} 9.0$ was utilized as antigen retrieval for PDGFR $\alpha$ and PDGFRß, whereas citrate buffer $0.01 \mathrm{M}$ pH 6.0 was employed for c-kit. The polymer system with DAB as the chromogen (Novocastra, Newcastle upon Tyne, UK) served as the detection system.

\section{Results}

We performed an expression study of the PDGFRA, PDGFRB and $K I T$ genes by RT-PCR and immunohistochemical analysis on 13 primary SBCs and on 1 recurrence from one of them (Fig. 1).

The immunohistochemical analysis revealed that the tumor stroma cells were positive for PDGFR $\alpha$ and PDGFRß expression in all the analyzed samples (Fig. 2).

In most cases, the neoplastic cells showed a focal expression of PDGFR $\alpha$ and PDGFRß (Fig. 2), mainly at the interface with the stromal component, while these receptors were found to be diffusely expressed in 4 and in 3 chordomas, respectively. PDGFR $\alpha$ and PDGFR $\beta$ were not observed to be expressed in the neoplastic cells of 2 and 1 chordomas, respectively. c-Kit immunoreactivity was detected in only one of the samples. The scattered mast cells served as the internal control in each case.

For the samples where immunohistochemical analysis was not feasible, we report data from the RT-PCR analysis (Fig. 1), which shows the transcripts of the three receptors in all but one of the samples, which lacks PDGFRB expression.

We also investigated by RT-PCR analysis the expression of the above TK receptor ligands ( $P D G F A, P D G F B$ and $S C F$ ), which might cause the receptor activation by means of an autocrine loop. In particular, $S C F$ was found to be expressed in only 1 tumor, while 8 chordomas showed both $P D G F A$ and $P D G F B$ mRNAs and 1 tumor showed only $P D G F B$ mRNA. The results are shown in Fig. 1.

\section{Discussion}

In the present study, we investigated the expression of the Imatinib target receptors, PDGFRA, PDGFRB and KIT transcripts and proteins, and of their ligands, $P D G F A, P D G F B$ and $S C F$ mRNAs, in 14 SBCs. The obtained evidence, indicating that all the analyzed samples express at least one of the three tyrosine kinase receptors under study and that 9 of them show the ligands $P D G F A$ and/or $P D G F B$ mRNAs, suggests the activated state of PDGFRs by means of an autocrine/paracrine loop. SCF mRNA was detected in one 


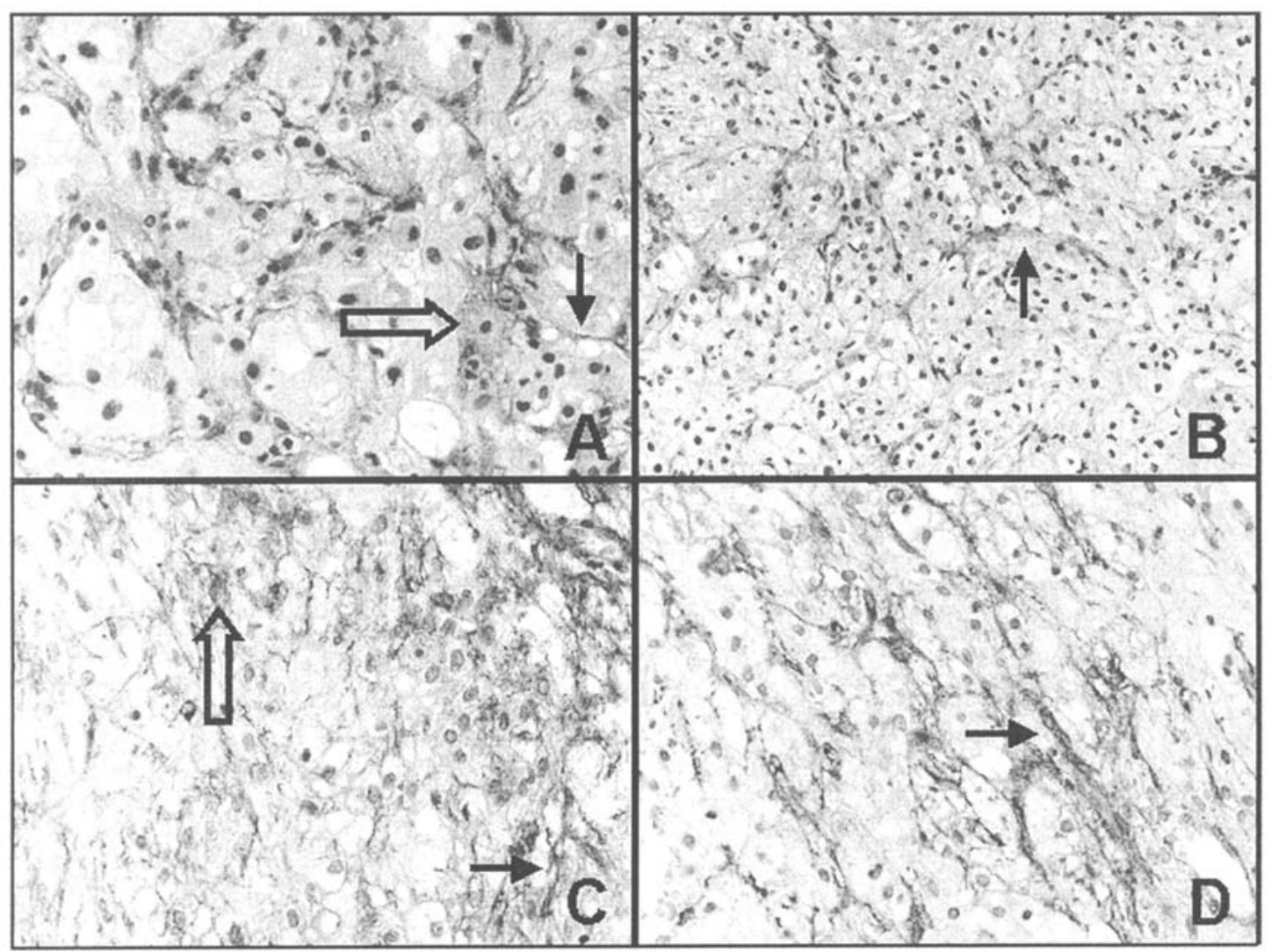

Figure 2. Immunohistochemical analysis showing (A) PDGFR $\alpha$ expression in the stromal and tumor cells of chordoma sample 12. (B) PDGFR $\alpha$ immunoreactivity was limited to the stromal component of chordoma sample 49. (C) PDGFRß expression in the stromal and tumor cells of chordoma sample 20. (D) PDGFRß is present mainly in the stromal cells with a more limited expression in the tumor cells of chordoma sample 21 . In A, C and D, the original magnification is $x 400$, in B x200. The open arrow indicates the neoplastic cells, and the black arrow indicates the stromal cells.

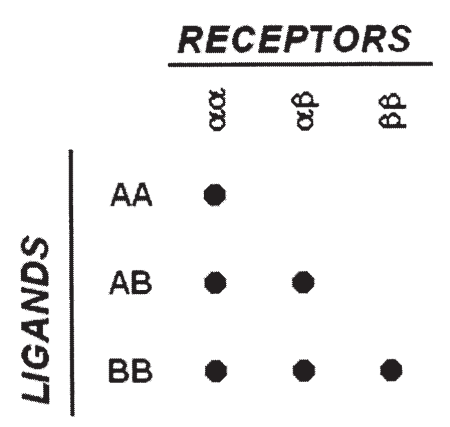

Figure 3. Schematic representation of the PDGFR $\alpha \alpha$, PDGFR $\alpha ß$ and PDGFRßß activation mechanism upon binding with their ligands, $P D G F A A, P D G F A B$ and $P D G F B B$. The black dots indicate the activated status of the receptors.

tumor. The lack of KIT expression though in the same tumor, excludes the biological role of c-kit activation in this instance.

The homodimeric or heterodimeric ligands activate different receptor dimers as shown in Fig. 3. It is therefore theoretically possible that 9 chordomas have an activated PDGFR $\alpha$ and/or PDGFRß.

The phosphorylated form of PDGFRß was detected in a sacral chordoma by Western blot analysis, while in the same study the presence of the receptor was postulated in five other samples, after the demonstration of $P D G F B$ gene expression (6).
Based on immunohistochemical analysis, we are the first to report on the cell-specific localization of PDGFR $\alpha$ and PDGFRß in SBCs. We observed that both tyrosine kinases are always expressed in the stromal component of the tumor tissue, while the neoplastic cells show a diffuse pattern of expression in only a few tumors. These findings suggest that the Imatinib mesylate pharmacological activity could be efficiently exerted in the stroma.

Stromal cells play essential roles in tumor determination: they constitute a solid substrate for tumor growth, are involved in tumor neo-angiogenesis and secrete growth factors (7).

The biological contribution of the stroma in the behavior of solid tumors and the significant stromal component in chordomas pinpoint a putative pharmacological action of Imatinib mesylate against chordomas. The reported colliquation of the spinal chordoma mass in a single case following the administration of Imatinib is consistent with the hypothesis of stromal plot necrosis. Casali et al describe the midterm signs of a decrease in tumor density on the CT scan and a change in the signal intensity on the MRI scan even without a noticeable impact on tumor size, which indeed can also increase (6).

This effect of Imatinib on the tumor mass could be dependent on the amount of the stromal component of the tumor and can lead to different consequences according to its localization. Tumor swelling in sacral chordomas does not usually lead to any serious or life-threatening clinical situations. 
An increase in tumor volume in clival chordomas could lead to the possible onset of new neurological deficits and eventual death from breathing failure due to the worsening of a preexisting brainstem compression. However, further observations on additional tumors are necessary in order to appreciate the effect of the treatment on chordomas.

Therefore, the above considerations suggest that the use of Imatinib could be indicated in the treatment of chordomas with specific localizations and carefully considered when a pre-existing compression of neurological vital structures has been assessed. Furthermore, its efficacy could be reduced in chordomas with a low stromal component. The response of the stromal component of chordoma, following the administration of Imatinib mesylate, should be delineated in further studies.

This study provides new insights into the localization of Imatinib targets in chordomas and contributes to addressing pharmacological and clinical protocols.

\section{Acknowledgements}

The authors thank Dr Michela Stroppi (Department of Biology and Genetics, Medical Faculty, University of Milan, Milan) for her valuable technical assistance. This study was supported by a 2005 grant from FIRST to P.R.

\section{References}

1. Crockard HA, Steel T, Plowman N, Singh A, Crossman J, Revesz T, Holton JL and Cheeseman A: A multidisciplinary team approach to skull base chordomas. J Neurosurg 95: 175-183, 2001.

2. Azzarelli A, Quagliuolo V, Cerasoli S, Zucali R, Bignami P, Mazzaferro V, Dossena G and Gennai L: Chordoma: natural history and treatment results in 33 cases. J Surg Oncol 37: 185-191, 1988.

3. Chugh R, Dunn R, Zalupski MM, Biermann JS, Sondak VK, Mace JR, Leu KM, Chandler WF and Baker LH: Phase II study of 9-nitro-camptothecin in patients with advanced chordoma or soft tissue sarcoma. J Clin Oncol 23: 3597-3604, 2005.

4. Scimeca PG, James-Herry AG, Black KS, Kahn E and Weinblatt ME: Chemotherapeutic treatment of malignant chordoma in children. J Pediatr Hematol Oncol 18: 237-240, 1996.

5. York JE, Kaczaraj A, Abi-Said D, Fuller GN, Skibber JM, Janjan NA and Gokaslan ZL: Sacral chordoma: 40-year experience at a major cancer center. Neurosurgery 44: 74-79, 1999.

6. Casali PG, Messina A, Stacchiotti S, Tamburini E, Crippa F, Gronchi A, Orlandi R, Ripamonti C, Spreafico C, Bertieri R, Bertuelli R, Colecchia M, Fumagalli E, Greco A, Grosso F, Olmi P, Pienotti MA and Pilotti S: Imatinib mesylate in chordoma. Cancer 101: 2086-2097, 2004.

7. Matei D, Emerson RE, Lai YC, Baldridge LA, Rao J, Yiannoutsos C and Donner DD: Autocrine activation of PDGFRalpha promotes the progression of ovarian cancer. Oncogene 25: 2060-2069, 2006.

8. Bhowmick NA, Neilson EG and Moses HL: Stromal fibroblasts in cancer initiation and progression. Nature 432: 332-337, 2004. 